\title{
The Possible Role of New Basal Insulin Analogues in Pediatric Population
}

\author{
Stefano Tumini, Flavia Amaro* \\ Department of Pediatrics, G. D'Annunzio University, Chieti, Italy
}

Received: May 16, 2018; Accepted: June 15, 2018; Published: June 22, 2018

*Corresponding author: Flavia Amaro, Department of Pediatrics, G. D’Annunzio University, Chieti, Italy, E-mail: flavia.amaro@hotmail.it

\begin{abstract}
In the pediatric age $\mathrm{HbA1c}$ values below $7.5 \%$ represent the ideal metabolic and glicemic target in DMT1 patients, but the literature shows that many of them are still very far from reaching this goal. The introduction of rapid and basal analogues, the reappearance of Continuous Subcutaneous insulin infusion (CSII), the progress in Continuous Glucose Monitoring (CGM) systems, the introduction of Sensor Augmented Pump (SAP), insulin pump system with Predictive Low-Glucose Management (PLGM) or Predictive Hyperglycemia and Hypoglycemia Minimization (PHHM) have brought about significant improvements in treatment options. The most promising new basal insulins are insulin degludec and insulin glargine U-300. The aim of this review is to analyze emerging data from the literature, also considering real-life data, in order to identify the clinical implication of the use of these new molecules.
\end{abstract}

\section{Introduction}

The achievement of the metabolic and glicemic target in DMT1 patients is a fundamental goal and, in the pediatric age, is represented by HbA1c values below 7.5\%. [1] The data of the literature show that this goal is far from being achieved.

The current basal-bolus schemes of insulin therapy are now very sophisticated but often there are economic, cultural and professional limitated resources for the education of patients and families and for the psycologic support to them.

Concerning the patient care, one of the most important problems is represented by the fear of hypoglycemia (FoH). [2] In this field, the introduction of rapid and new basal analogues, the reappearance of Continuous Subcutaneous insulin infusion (CSII), the progress in Continuous Glucose Monitoring (CGM) systems, the introduction of Sensor Augmented Pump (SAP), insulin pump system with predictive low glucose management (PLGM) have improved the DM management. [3]

The aim of this review is to analyze the potential role of the new insulin analogues, also considering real-life data in order to light up the strategies for improvements in the use of these drugs in daily clinical routine.

\section{Which is an ideal basal analogue?}

The basal bolus model of insulin therapy reproduces the endocrine pancreatic secretion of insulin, which is bimodal: a basal insulin secretion which covers fasting periods and counteracts gluconeogenesis and ketogenesis and the secretion of insulin at mealtimes.

An ideal basal insulin should have the following characteristics: peakless, a low risk for hypos, a long flat profile of action, a predictable profile of pharmacodynamic (Pd) effects with limited interand intra-individual variability; it should be also highly tolerable, low cost and without signals of increased mitogenicity; it should also limit weight gain in users. Other important characteristics are: duration of action longer than 24 hours, a flat peakless profile with homogeneous distribution of the glucose-lowering effect during the day and with a low intrasubject variability across a dosing interval and from day to day.

Thanks to all these characteristics of a basal insulin, it could be easier to get the goal of optimal HbA1c and it could be possible to try to improve one of the most important issues of insulin therapy: fear of hypoglycemia.

\section{New basal analogues}

\section{Insulin degludec}

Insulin degludec (IDeg) (Novo Nordisk A/S, Bagsvaerd, Denmark) is a new analogue with ultra-long duration of action. It has a half-life longer than 25 hours. It gets steady state after 2-3 days in subjects with T1DM. It is characterized by a first-order kinetics. In subcutaneous depot IDeg forms multi hexamers polymers from which single monomers gradually break off and reach the bloodstream. [4]

\section{Insulin glargine $\mathrm{U} 300$}

The most recent clinical approach for ultra-long insulin is glargine U300 (300 U/mL) (IGlar-U300) (Sanofi, Frankfurt, Germany). Exposure to U300 is more evenly distributed, and glucose control remains for 36 hours, resulting in decreased hypoglycemic episodes.[5] 


\section{Pharmacokinetic and pharmacodynamic properties of new basal analogues}

The actual basal analogues are characterized by day-to-day "peaks and valleys" and so also by a rigid timing of administration. The new basal insulin with a duration of action longer than 24 hours tends to have lower day-to-day variability.[6]

\section{Variability factors of IDeg and IGlar-U300}

Beyond the pharmacodynamic and the pharmacokinetic properties, another crucial issue is the correct family and patient education to reduce variability factors like the correct use and the quality of the device, the right preservation, the right time to wait before needle extraction, the appropriate needle length considering the subcutaneous tissue thickness, the control of the injection site in order to avoid lip hypertrophies.[7]

\section{BEGIN@ and EDITION $®$ Program (phase IIIa/IIIb studies about IDeg and IGlar)}

\section{Insulin degludec BEGIN $®$ Program: study in TDM and in T2DM}

The insulin degludec efficacy has been evaluated through the BEGIN@ program (Novo Nordisk a/S, Bagsvaerd, Denmark): it is made of some studies that are randomized, open-label and treatto-target and they are non-inferiority trial. IDeg has been noninferior compared to IGlar about HbA1c decreasement. Besides, patients with IGlar had an incidence of nocturnal hypoglycemia higher than 25\% compared to those treated with IDeg (target of FPR: $70-89 \mathrm{mg} / \mathrm{dL}$ ).[8] The conclusion of a study in adults is that a similar achievement in HbA1c is possible with IDeg vs. IGlar with a lower rate of hypoglycemic episodes. $[9,10]$

\section{Insulin glargine EDITION@ Program: study in T1DM and in T2DM}

EDITION® is an international program of phase III about the efficacy and safety of IGlar-U300 (Sanofi, Frankfurt, Germany) compared to IGlar-U100. In DMT1 patients IGlar-U300 is non inferior about HbA1c target with a reduction of hypoglycemic episodes (also of nocturnal ones). In DMT2 patients there are similar HbA1c control and a similar rate of adverse events (AEs) and hypoglycemia.[11] A study about pediatric age is currently underway (EDITION $®$ JUNIOR-ClinicalTrials.gov, ID: NCT02735044).

\section{Clinical implications in pediatric patients}

\section{Hypoglycemia}

IDet and IGlar are basal insulin analogues that have shown a longer duration of action, a reduced variability and a decreased risk of hypoglycemia, particularly the nocturnal one, compared to neutral protamine Hagedorn (NPH).[12] In this way, they can help to get HbA1c target without increasing the hypoglycemic episodes, that is fundamental in pediatric patients.

Concerning IDeg and IGlar-U300, only data about IDeg are available in pediatric age.[10] Compared to other long acting insulin analogues IDeg is non-inferior for $\mathrm{HbA1c}$ reduction, but superior for lower fasting plasma glucose. IDeg is associated with lower insulin dosing, reduced nocturnal hypo rate and has a similar number of AEs, but we need more studies concerning youth with DMT1.[13] In any case the few studies that we have about pediatric patients seems to confirm the data of the studies about adults. [13]

\section{Ketosis prevention}

The data from a recent meta-analysis of BEGIN@ pediatric trial (ClinicalTrials.gov, ID: NCT01513473) show reduced rate of hyperglycemia of IDeg for ketones levels $>0.6,>1.5$, and $>3.0$ $\mathrm{mmol} / \mathrm{L}$ compared with IDet.[10,13] IDeg could potentially prevent hyperglicemia with ketosis in pediatric patient compared to IDet.

\section{Basal insulin titration}

The reduced variability of new basal insulin could help to get a fine titration of basal insulin without increasing the risk of nocturnal hypoglycemia, also through the use of CGM and with Flash Glucose Monitoring (FGM). FGM is a recently introduced promising system and a technologically advanced device that shows most of the advantages of CGM.[14]

\section{Basal insulin doses in real life}

There are no evidence-based pediatric guidelines about the ideal determination of basal needs that are specific for age, gender, pubertal stage or the disease stage. The studies that have been carried out during fasting show a $0.2 \mathrm{U} / \mathrm{Kg} /$ die requirement but they have been carried out in a non-physiological status and, in many cases, with patients with CSII therapy.[15,16] In any case, it could be possible to think that using new basal insulin in patients not used to apply the algorithm based on flexible carbohydrate counting and starting blood glucose level, cannot improve the metabolic control or may even make it worse.

\section{Fear of hypoglycemia}

A long-acting basal insulin analogue with a flat, stable absorption profile could reduce nocturnal hypoglycemia and FoH in adults. Because of hypoglycemic episodes, parents and children can develop unsuitable behaviors like the sleeping disruption. Often they accept too high glycemic target (with relative reduction of insulin doses) when children are not under the direct parents' control. $[17,2]$ The new technologies about the insulin bolus calculation and the glucose blood level monitoring allow a reduction of the FoH. The CGM impact on the FoH is not so clear. The use of new basal analogues has to be integrated with the new technologies.

\section{QoL and treatment satisfaction}

The impact on health-related QoL (HrQoL) was assessed only in adult patients. All the studies show that hypoglycemia and $\mathrm{FoH}$ are main components of HrQoL in some patients but also glycemic variability and unpredictability have a significant role in determining HrQoL. [18] New studies about FoH and the consequence on HrQol in the pediatric age are necessary.

\section{A more flexible time scheme administration}

Concerning the practical problems about the compliance 
of insulin therapy, there is the necessity to administer insulin at regular times. The new basal insulins, thanks to their long duration, allow the avoidance of a rigid time scheme for insulin administration.[19] Flexibility in insulin administration in well educated patients and families is probably one of the most important gains in terms of HrQoL, so clinical studies in this field will be very important.

\section{Physical activity management}

There are no guidelines about physical activity management in patients who use IDeg. The decrease of the insulin dose at least 48 hours before the activity is not recommended.[20]

\section{Injection site}

The flat pharmacokinetic and Pd profiles would be less susceptible to the small differences in the absorption rate and the differences in absorption rate among the possible injection sites are not clinically relevant.[21]

\section{How to manage IDeg in real-life}

The pediatric data about pharmacokinetics, safety and efficacy of IDeg, obtained through clinical trials, should be shifted in the daily practical use. There are specific types of pediatric patients who can benefit from the use of IDeg (to date the only one analogue of last generation approved for age from 1-18 years in Italy): patients who present recurrent hypoglycemia episodes and whose FoH doesn't allow to reach the glicemic target; patients who don't achieve glycemic targets with the basal insulin already used and so who could have a better compliance through a greater flexibility in administration times; patients who need a twice-daily (TD) analogue administration or present a marked dusk or dawn phenomenon using other analogues. Before switching to IDeg is necessary a preparatory supportive training focusing on the basal bolus therapy. In particular, it should be clearly understood how much the basal analogue affects the nocturnal glycemic trend. It is essential first to know the bedtime blood glucose levels to achieve a correct IDeg dose titration (with a greater number of Self Monitoring Blood Glucose -SMBGs- or, when possible, a correct interpretation of CGM data together with SMBGs). For the initial dose of IDeg, it is necessary to pay attention particularly to those patients who have a basal need $>0.5 \mathrm{U} / \mathrm{Kg} /$ die or are used to do TD administration; it is also important to consider that steady state is reached in the third-fourth day of therapy. In many cases 10\% reduction is appropriate, except for patients who administered IDet or IGlar TD: in this case $20 \%$ reduction is appropriate; concerning patients who aren't on target about HbA1c, the dose of the basal insulin could remain the same. In any case, the initial dose requires a high degree of individualization, considering the level of self-management education, the compliance with SMBG, the possible effective use of CGM, the willingness to keep in adequate contact with the diabetologist (also a telephone contact).[22]

Since the IDeg profile is flat over 24 hours, it is reasonable to think about a change of the doses of pre-prandial rapid analogue. The decline in action of the previous basal analogues in the final part of the daily profile means that, after introducing IDeg, the dose of the rapid analogue should be reduced for the meals consumed during the phase of basal hypo-insulinization (e.g. breakfast in patients who administer IGlar at lunchtime or at breakfast-time). When possible, these families and patients are persuaded to use the titration algorithm based on CHO counting, after a suitable education. Also the insulin-CHO ratio and the insulin sensibility factor are redefined before starting the therapy with IDeg on the basis of the previous considerations and the parameters advised according to age and stage of puberty. [22]

\section{Conclusions}

It could be desiderable a proactive approach to establish which patients can benefit from the new analogues but also to optimize their use. Currently, there is a big gap about the real-life data. A correct educational "strategy" is essential to personalize the care plan and to reach the glycemic target. The education programs must take into account the specific cultural, anthropological, psychological aspect and, in children and adolescents, the level of cognitive development, the stage of growth and family situations. $[1,23]$

\section{References}

1. Chiang JL, Kirkman MS, Laffel LMB, Peters AL. Type 1 diabetes through the life span: A position statement of the American Diabetes Association. Diabetes Care. 2014;37:2034-2054. Doi: 10.2337/dc141140

2. Driscoll KA, Raymond J, Naranjo D, Patton SR. Fear of Hypoglycemia in Children and Adolescents and their Parents with type 1 Diabetes. Vol. 16, Current Diabetes Reports. 2016;16(8):77. Doi: 10.1007/ s11892-016-0762-2

3. Choudhary P, Olsen BS, Conget I, Welsh JB, Vorrink L, Shin JJ. Hypoglycemia Prevention and User acceptance of an Insulin Pump System with Predictive Low Glucose Management. Diabetes Technol Ther 2016;18(5):288-291. Doi: 10.1089/dia.2015.0324

4. Haahr H, Heise T. A review of the pharmacological properties of insulin degludec and their clinical relevance. Clin Pharmacokinet 2014;53(9):787-800. Doi: 10.1007/s40262-014-0165-y

5. Becker RHA, Dahmen R, Bergmann K, Lehmann A, Jax T, Heise T. New insulin glargine 300 Units $\cdot \mathrm{mL}-1$ provides a more even activity profile and prolonged glycemic control at steady state compared with insulin glargine 100 Units $\cdot \mathrm{mL}-1$. Diabetes Care. 2015;38(4):637-643. Doi: 10.2337/dc14-0006

6. Heise T, Hermanski L, Nosek L, Feldman A, Rasmussen S, Haahr H. Insulin degludec: four times lower pharmacodynamic variability than insulin glargine under steadystate conditions in type 1 diabetes. Diabetes Obes Metab 2012;14(9):859-864. Doi: 10.1111/j.1463-1326.2012.01627.x

7. Gin H, Hanaire-Broutin H. Reproducibility and variability in the action of injected insulin. Diabetes Metab 2005;31(1):7-13.

8. Heller S, Buse J, Fisher M, Garg S, Marre M, Merker L, et al. Insulin degludec, an ultra-longacting basal insulin, versus insulin glargine in basal-bolus treatment with mealtime insulin aspart in type 1 diabetes (BEGIN BasalBolus type 1): a phase 3, randomised, open-label, treatto-target non-inferiority trial. Lancet 2012;379(9825):1489-1507. Doi: 10.1016/S0140-6736(12)60205-0

9. Ratner RE, Gough SCL, Mathieu C, Del Prato S, Bode B, Mersebach 
$\mathrm{H}$, et al. Hypoglycaemia risk with insulin degludec compared with insulin glargine in type 2 and type 1 diabetes: a pre-planned metaanalysis of phase 3 trials. Diabetes Obes Metab 2013;15(2):175-184. Doi: 10.1111/dom.12032

10. Thalange N, Deeb L, Iotova V, Kawamura T, Klingensmith G, Philotheou $\mathrm{A}$, et al. Insulin degludec in combination with bolus insulin aspart is safe and effective in children and adolescents with type 1 diabetes. Pediatr Diabetes 2015;16(3):164-176. Doi: 10.1111/ pedi.12263

11. Home PD, Bergenstal RM, Bolli GB, Ziemen M, Rojeski M, Espinasse $\mathrm{M}$, et al. Glycaemic control and hypoglycaemia during 12 months of randomized treatment with insulin glargine $300 \mathrm{U} / \mathrm{mL}$ versus glargine $100 \mathrm{U} / \mathrm{mL}$ in people with type 1 diabetes (EDITION 4). Diabetes Obes Metab. 2018;20(1):121-128. Doi: 10.1111/dom.13048

12.Bartley PC, Bogoev M, Larsen J, Philotheou A. Longterm efficacy and safety of insulin detemir compared to Neutral Protamine Hagedorn insulin in patients with Type 1 diabetes using a treat-totarget basal-bolus regimen with insulin aspart at meals: a 2-year, randomized, controlled trial. Diabet Med. 2008;25(4):442-449. Doi: 10.1111/j.1464-5491.2007.02407.x

13. Mathews C, Gallagher K, Acerini CL. A comparative, systematic, meta-analysis of the safety and efficacy of insulin degludec (IDeg). Does IDeg confer any advantage over other long-acting analogues in young patients with type 1 diabetes? 42nd ISPAD Annual Meeting. Pediatr Diabetes 2016;17(Suppl. 24):106.

14. Rodbard D. Continuous Glucose Monitoring: a review of Successes, Challenges, and Opportunities. Diabetes Technol Ther. 2016;18(Suppl. 2):S23-S213. Doi: 10.1089/dia.2015.0417

15. Danne T, Bangstad H-J, Deeb L, Jarosz-Chobot P, Mungaie L, Saboo B, et al. ISPAD Clinical Practice Consensus Guidelines 2014. Insulin treatment in children and adolescents with diabetes. Pediatr Diabetes. 2014;15(Suppl 2):115-134. Doi: 10.1111/pedi.12184
16. Kuroda A, Kaneto H, Yasuda T, Matsuhisa M, Miyashita K, Fujiki N, et al. Basal insulin requirement is $\sim 30 \%$ of the total daily insulin dose in type 1 diabetic patients who use the insulin pump. Diabetes Care 2011;34(5):1089-1090. Doi: 10.2337/dc10-2149

17. RE G, Fulcher G. Reduction in fear of hypoglycemia in subjects with sub-optimally controlled diabetes using a glargine insulinbased treatment regimen. Can J Diabetes. 2008;32(2):101-106. Doi: 10.1016/S1499-2671(08)90000-9

18.Shi L, Shao H, Zhao Y, Thomas $\mathrm{N}$ a. Is hypoglycemia fear independently associated with health-related quality of life? Health Qual Life Outcomes 2014;12:167. Doi: 10.1186/s12955-014-0167-3

19. Peyrot M, Barnett AH, Meneghini LF, Schumm-Draeger PM. Insulin adherence behaviours and barriers in the multinational Global Attitudes of Patients and Physicians in Insulin Therapy Study. Diabet Med. 2012;29(5):682- 689. Doi: 10.1111/j.1464-5491.2012.03605.x 20. Riddell MC, Gallen IW, Smart CE, Taplin CE, Adolfsson P, Lumb AN, et al. Exercise management in type 1 diabetes: a consensus statement. lancet Diabetes Endocrinol 2017;5(5):377-390. Doi: 10.1016/ S2213-8587(17)30014-1

21. Nosek L, Coester H-VV, Thomsen HF, Roepstorff C, Haahr HL, Sin R, et al. Glucose-Lowering Effect of Insulin Degludec is Independent of Subcutaneous Injection Region. Clin Drug Investig 2014;34(9):673679. Doi: 10.1007/s40261-014-0218-x

22.Tumini S, Carinci S. Unmet needs in children with diabetes: the role of basal insulin. Minerva Pediatr. 2017;69(6):513-530. Doi: 10.23736/S0026-4946.17.05112-X

23. Haas L, Maryniuk M, Beck J, Cox CE, Duker P, Edwards L, et al. National standards for diabetes self-management education and support. Diabetes Care 2012;35(11):2393-2401. Doi: 10.2337/dc121707 